\title{
Challenges and Prospects in the Provision of Counseling Services for Art Patients: The Case of Nekemte Referral Hospital, East Wollega, Oromia
}

\author{
Abdissa Gemechu Gobena* \\ Department of Psychology, Nekemte College of Teacher Education, Ethiopia
}

Submission: October 21, 2019; Published: November 13, 2019

*Corresponding author: Abdissa Gemechu Gobena, Department of Psychology, Stream of Education, Nekemte College of Teacher Education, Post Box No. 88, Nekemte, Ethiopia

Abstract

This study was conducted with the main objective of describing the challenges and prospects in the provision of counseling services for ART patients who were attending their follow up at Nekemte Referral Hospital chronic illness clinic. A descriptive qualitative study design was used to collect qualitative data from study participants (40 ART patients attending in chronic illness follow up clinic and 8 service providers) from March to July 2017. Nonprobability and purposive sampling techniques was used to select participants available during the study period. Data were collected by trained data collectors through face to face conversation for 25-30 minutes using un-structured interview questioner was first prepared in English and translated into Afan Oromo. The data gathered in Afan Oromo and transcribed, then translated back to English for analyses. The translated data were identified line by line and taken in general senses; categorized into data-based themes that emphasized by the respondents actual words and used for analysis in the narrative form. The results of the study were presented in words in the narrative form. In relation to Challenges socio-demographic characteristics related, service providers related ,clients related ,service related and psychosocial related problems were described as major problems that might resulted in common challenges including poor relationships between service providers and clients, poor or non-systematic flow of information during counseling provision ,confidentiality and long waiting time problems. In connection with Prospects ART patients negative interests /beliefs/negative prospects in participating in both medications and treatments processes which might resulted from the clients poor satisfaction due to challenges in the provision of counseling services. The implication of this study finding for future research into challenges and prospects in the provision of counseling services for ART patients are suggested and data based conceptual frameworks are constructed.

Keywords: Counseling Services; ART Patients; Service Providers; Challenges; Prospects

Abbreviations: HIV: Human Immune Deficiency Virus; AIDS: Acquired Immune Deficiency Syndrome; ART: Anti-Retroviral Treatment; PLWHIV: People Living with HIV; HRQoL: Health Related Quality of Life; WHO: World Health Organization; QoL: Quality of Life; MoH: Ministry of Health; HTC: HIV Testing and Counseling; VCT: Voluntary Counseling and Testing; NRH: Nekemte Referral Hospital; VC: Voluntary Counseling; DC: Data Collector; PI: Principal Investigator

\section{Historical Background}

Throughout history, the world has never faced a health challenges like HIV/AIDS Pandemic. The advent of HIV/AIDS has brought many challenges to health systems in both developed and developing countries. One such challenge is the need to provide adequate health care to the increasing number of people who become ill as a result of HIV infection. In order to solve such problems, various types of services exist to provide health care to People Living with HIV/AIDS (PLWHA) that might include the role of home care and other community-oriented care services has gained prominence with the shift in the character of HIV/ AIDS-related illness from sub-acute to largely chronic one.
According to Ritzenthaler [1], the global community cited ART as a key component of effective HIV/AIDS Programs. In their declaration of commitment, heads of state from 189 countries affirmed that prevention, care, support and treatment for those infected and affected by HIV/AIDS are mutually reinforcing elements of an effective response and must be integrated in a comprehensive approach to combat the epidemic.

HIV/AIDS is progressive, lifelong condition that is managed with lifestyle changes and medications resulted from counseling to prevent the distribution of HIV/AIDS and the problems of HIV/AIDS on Anti-Retro viral (ART) patients. The problems 
are characterized by an increase in individual patients (clients) personal, physical, psychological, emotional, social and economic contexts WHO [2]. On the other hand, good adherence to ART treatment increases better clinical outcomes. To do so, it has been recommended that the stepping-stones towards the achievement of long-term virus control among patients in developing countries are simplification of therapy, improvement of patient adherence and minimization of drug resistance [3].

Trust is the most important factor in the counselor-client relationship which enhances the relationship and improves the odds that an individual will act decisively on the information provided. Given the discrimination, ostracism, and personal recrimination that an individual diagnosed with HIV may have to face, it is all the more important to guarantee confidentiality [4]. Therefore, the advent of potent antiretroviral treatment (ART) has dramatically reduced rates of mortality and morbidity, improved quality of life, revitalized communities and transformed perceptions on HIV/AIDS from a plague to a manageable chronic illness [5]. Coming to Africa, specifically, the Sub-Saharan Africa is disproportionately affected by the HIV/ AIDS pandemic. With about $10 \%$ of the world's total population, Sub-Saharan Africa accounted for three quarters of AIDS deaths worldwide and up to two-thirds of all people living with HIV/ AIDS.

In Ethiopian context, the government issued a national policy on HIV/AIDS in 1998. The policy aims to direct the various efforts put forth to mitigate the impact of the pandemic. As a result, currently, achieving and maintaining acceptable levels of quality of care in the view of decentralization and rapid scale up of HIV prevention, care and treatment in Ethiopia is a matter of concern, despite providing good quality health care to the increasing number of PLWHIV is a great challenge mainly due to lack of resources, and professionals' work shift. It is recommended that even though many factors govern HIV service quality, a well-designed and coordinated clinical mentoring process is taken as an immediate option of interventions to achieve the desired quality of HIV care/ART [6].

According to East Wollega Development Association [7] the health report in the study indicated that HIV $\backslash$ AIDS is a big threat for East Wollega Zone, Southern Ethiopia in Referral like any other areas in the country, and Nekemte Referral Hospital in particular. Besides, the report described that HIV AIDS is crippling the potential and morale of the youth, who are expected to contribute significant role in the country's development process. The report had also described the causes and effect of HIV $\backslash$ AIDS in the study area as seasonal migration to the neighboring zones \areas, polygamy, rape, and abductions and lack of awareness creation on its mode of transmission and other Referral local level stigma and discrimination are the major exposing factors to the pandemic in East Wollega Zone.

In Referral, based on the above information and absence of published studies and information gaps experienced in the study area initiated the researcher to describe the challenges and prospects in the provision of counseling services for ART patients who were attending their follow ups in the Nekemte Referral Hospital by considering the study participants' behaviors, experiences, opinions, values, feelings, emotions, knowledge and socio-demographic question types.

\section{Statements of the Problems}

HIV/AIDS is a major source of concern all over the world as it constitutes a major source of death and a threat to national development since the virus has negative impacts on economic, social and political development of any nation that has its high rate [8]. Because of this pandemic and morality nature of HIV/ AIDS, now a day, public health officials assumed that its spread could be halted by informing people how to protect themselves from infection and by safeguarding blood supplies [1].

In order to define the challenges and identify the solutions, both HIV/AIDS counseling and testing aim at assisting patients to understand themselves, adjust effectively to life challenges, and contribute meaningfully to the development of the society. Furthermore, Voluntary Counseling and Testing (VCT) is a key element to identify HIV infected persons who could benefit from therapeutic interventions [9]. In doing so, diagnosing and treating health problems such as depression, reducing substance abuse, improving patient and provider relationship, counseling, and enhancing family and community support mechanisms are shown to improve adherence as well as intervening on modifiable barriers to adherence before starting ART [10].

In relation to in patient-centered health care, the first challenges are to identify and meet the changing needs of patients. So that service providers need to ensure that people can access counseling or medications easily and as far as possible in away and at a time and place of their own choice. They can also empower patients engaging herein dialogue to communicate knowledge which enables them to manage their own health and treatment. In addition, using health care interventions can no longer be based on opinion or individual experience alone rather it needs integration among service providers [11]. So, it is obvious that good health is an essential condition to meaningful national development. HIV/AIDS is a threat to life, thus individuals need to know their HIV status through testing since such a test helps to reduce transmission and involvement in risky sexual behaviors as well as it promotes early treatment and adjustment [6].

In the late 1980s, as the epidemic surged and shifted from people with high-risk behavior to the Referral population, especially in developing countries, so that Ritzenthaler [1] recommended that public health professionals should realize that they needed to better understand the causes of individual infection and the forces driving the pandemic that help health professionals to know how HIV transmission was linked to specific behavior such as having multiple sex partners, 
commercial sex, and injecting drug use, but they also realized that these behaviors were influenced by political, economic, social, cultural, and environmental factors-including poverty, military conflict, powerlessness, and gen .Providing counseling to clients with HIV/AIDS involves educating a client or a group of clients on the control, management and prevention of HIV/AIDS. That is counseling assists clients to make informed decisions, cope better with life challenges, lead positive lives and prevent further transmission of HIV [8].

One study finding, in Ethiopian context, showed that the provision of counseling services for ART patients do not exist in isolation from other health care services. Rather it should be collaborated with patients, counselors, physicians, nurses, midwifes and other health care providers. Thus service providers, together with ART patients, are responsible directly to patients for every issue under treatment processes in order to have a positive outcome on ART patients' quality of life [12]. These authors identified the major challenges such as the knowledge gap in pediatric HIV and the lack of counseling skills, as well as health system which were reported in the delivery of pediatric HIV services [13]. In any health care services, including HIV/AIDS counseling, client satisfaction is an important component when measuring health outcomes and quality of care. For instance, satisfied clients are more likely to develop a deeper and longer lasting relationship with their health care providers, leading to improved compliance, continuity of care, and ultimately better outcomes [14].

To sum up, study reports across the world showed that ART patients dropped out and interruption of both medication and counseling services which resulted in decrease ART patients' quality of life and high hospital admission. However, despite HIV \AIDS is a sensitive issue and major public health problem in Ethiopia including in the study area, as to the knowledge of the researcher, there is no study done on challenges and prospects in the provision of counseling services for ART patients. Therefore, this information gap drove the researcher to conduct this study. Accordingly, the study described the challenges and prospects in the provision of counseling services for ART patients in Ethiopia, especially in Nekemte Referral Hospital and constructed data based conceptual frameworks that could help to ART patients, HIV counseling service providers, future researchers and policy makers.

On its course, the study answered the following basic research questions:

a) What were the major challenges in the provision of counseling service for ART patients?

b) What were the common challenges in the provision of counseling services for ART patients?

c) How did the respondents describe their satisfaction in contexts of the challenges and prospects in the provision of counseling services for ART patients? d) How did the respondents describe their prospects in the provision of counseling services for ART patients?

\section{Objective of the Study}

The general objective of this study was to describe the Challenges and Prospects in the provision of counseling services for ART patients attending chronic illness follow up at Nekemte Referral Hospital. To fulfill the general objectives, this study involved the following specific objectives to:

i. Identify the major challenges in the provision of counseling services for ART patients in NRH.

ii. Identify the common challenges in the provision of counseling services for ART patients in NRH.

iii. Asses and describe respondents' satisfaction in the context of the challenges in the provision of counseling services for ART patients in NRH.

iv. Identify and describe the respondents' prospects in the provision of HIV counseling services in participation of their treatments.

\section{Significance of the Study}

It is obvious that, no researches have been conducted with the same inquiry of this research in Ethiopia, particularity in Nekemte referral hospital. This study was conducted the qualitative research on the challenges and prospects in the provision of counseling services for ART patients. Therefore, it was significant since it was identified and described problems that might face in the Ethiopian hospitals ART patients who were attending their follow up care in the hospital. Furthermore, this study was believed to open ways to other researchers who are interested on issues related to this work of research. Finally, the constructed data based conceptual frameworks and forwarded recommendations on challenges and prospects in the provision of counseling services for might give important clues for all ART patients, service providers and to other concerned bodies and organizations including the government.

\section{Literature Review}

\section{Overview of HIV/AIDS}

Patients living with HIV (PLWHIV) may experience reduced independence and ability to undertake certain activities of daily living, as well as psycho social, and economic incapacity. Since, HIV/AIDS is a chronic illness condition; HIV counseling (treatment) targets progression of the disease, improvement of prognoses and reduction of challenges up on ART patients.

\section{Challenges in the provision of counseling for ART patients}

This study involved descriptive qualitative study, the selected studies in the literature review included both quantitative studies (to identify previous research findings and directions) 
and qualitative studies that had helped to understand the experiences of both ART patients and service providers on the challenges and prospects in the provision of counseling services for ART patients and information from theories books reports had helped to describe challenges in the current study. Accordingly, the challenges in the provision of counseling for ART patients include under the literature involved lack of integration among service providers, lack of cooperation among community and family, poor relationship of clients within families and societies and between the clients and service providers, ART client's personal, interpersonal, emotional, psychological and economic conditions; medication side effects, social influences, attitude, knowledge gaps, awareness, resistance, sociodemographic related problems, resource limitation, professional work shifts, privacy, confidentiality, poor adherence and health related problems. The literature review had covered not all but some selected studies and information from theories and books and reports associated with challenges and prospects in the provision of counseling services for ART patients in Referral and with this study findings in particular.

The HIV/AIDS pandemic has become a health, socialeconomic and development disaster, with far reaching and implication for individuals, communities and countries. No other disease has so dramatically highlighted the current disparities and inequities in the health care access, economic opportunity and the protection of basic human right [15]. With this regard, AIDS has brought many challenges to health systems in both developed and developing countries. One such challenge is the need to provide adequate health care to the increasing number of people who become ill as a result of HIV infection. Various types of services exist to provide health care to people living with HIV/AIDS (PLWHA) including the role of home care and other community-oriented care services has gained prominence with the shift in the character of HIV/AIDS-related illness from sub-acute to largely chronic.

On the other hand, HIV/AIDS is progressive, lifelong condition that is managed with lifestyle changes and medications resulted from counseling services to prevent the distribution of HIV/AIDS and the problems of HIV/AIDS on ART patients. The problems are characterized by an increase in individual patients (clients) personal, physical, physiological, emotional, social and economic contexts [2]. According to the report of Global HIV/ AIDS (2004), about HIV/AIDS challenges to health systems in both the developing and developed countries had indicated that the advent of HIV/AIDS has brought many challenges to health systems in both developed and developing countries. One such challenge is the need to provide adequate health care to the increasing number of people who become ill as a result of HIV infection. Various types of services exist to provide health care to PLWHA. The role of home care and other community-oriented care services has gained prominence with the shift in the character of HIV/AIDS-related illness from sub-acute to largely chronic. In patient centered health care the first challenges are to identify and meet the changing needs of patients. Service providers need to ensure that people can access counseling or medications easily and as far as possible in away and at a time and place of their own choosing. They can also empower patients engaging herein dialogue to communicate knowledge which enables them to manage their own health and treatment for using health care interventions can no longer be based on opinion or individual experience alone [11].

Other qualitative studies from Botswana, Tanzania and Uganda showed that although patients are highly motivated to take ARVs as prescribed, constraints such as transport costs, user fees, long waiting times, hunger, stigma, side-effects and lack of appropriate counseling undermine their intentions to do so.

On the other hand, study conducted by Dan \& Fauci [16] revealed that the key factors of sub-optimal adherence to ART, in developed countries, are patient and treatment related, including substance and alcohol abuse, complexity dosing regimen and 'pill burden', dietary restrictions and side effects .In contrast the study conducted by Holmberg [5] showed about the effect of ART that the advent of potent antiretroviral treatment (ART) has dramatically reduced rates of mortality and morbidity, improved quality of life, revitalized communities and transformed perceptions on HIV/AIDS from a plague to a manageable chronic illness. Available evidence indicated that HIV/AIDS counseling involves educating a client or a group of clients on the control, management and prevention of HIV/AIDS. Counseling assists people to make informed decisions, cope better with life challenges, lead positive lives and prevent further transmission of HIV. Diagnosing and treating health problems such as depression, reducing substance abuse, improving patient and provider relationship, counseling and enhancing family and community support mechanisms are shown to improve adherence, as well as intervening on modifiable barriers to adherence before starting ART [10].

Similarly, Assefa and kloos had conducted a research on antiretro-viral therapy (ART) in middle and low income countries. This study reported the need to maintain achieve equitable access, failure reach those who haven't known their HIV status, and difficulty of convincing people living with HIV and AIDS who are reluctant to receive therapy until they see AIDS related illness, are some of the challenges observed along the expansion of ART. The study added that poor adherence (often leading to drug a resistance), complete discontinuation of the treatment, some long term and short-term side effects resulting from ART management, are other key problems occurring following ARV therapy.

Regarding the factors hindering acceptance of HIV/AIDS by ART patients, Okpoto [11] identified such factors as ignorance, fear of being positive, cost of VCT, inadequate number of VCT centers and stigmatization that depend on and available to the respondents as regards to the experience of HIV/AIDS 
patients . In the study ignorance, fear of being positive, cost of VCT, inadequate number of VCT centers and stigmatization constituted major hindrances to acceptance of HIV/AIDS VCT. The author further recommended that more awareness should be created among rural youths on the need for HIV/AIDS VCT and suggested that HIV/AIDS requires collaborative efforts in view of its devastating effects and people should be encouraged to obtain information about their HIV status and seek prompt counseling and medical intervention.

A team of South African researchers carried out a study on factors associated with participation in HIV voluntary counseling and testing among TB patients in a rural South African hospital. In the study, demo-graphic characteristics, knowledge, attitudes and beliefs regarding HIV/AIDS were measured in order to determine possible associations with the decision to accept or reject VCT. Achieving and maintaining acceptable levels of quality of care in the view of decentralization and rapid scale up of HIV prevention, care and treatment in Ethiopia is a matter of concern. But providing good quality health care to the increasing number of PLHIV is a great challenge mainly due to lack of resources and professional work shift. Even though many factors govern HIV service quality, a well-designed and coordinated clinical mentoring process is taken as an immediate option of interventions to achieve the desired quality of HIV care/ART [13].

Rosen \& Gill [3] conducted a research under retention of antiretroviral therapy in the context of sub Africa. In the study, age, income and duration after diagnosis had significant relationship with HRQOL when analyzed individually in binary logistic regression analysis but were not significant when entered into multi variable logistic regression. From socio demographic characteristic; sex, marital status, religion, ethnicity and place of residence all did not had significant statistical relationship with HRQOL of the patients even in binary logistic regression analysis.

As Sibhatu \& Ayalu [17] conducted their researches by using over 100 articles and abstracts written in English, which in turn led to analyses of works reviewed under three thematic headings that are adherence estimates, adherence barriers, and suggested solutions for mitigating the problem. The researchers in their study had shown ancestries of challenges and problems resulting from poor adherence that are categorized under patients and family related challenges, socio-economic and environmental challenges, medication related challenges, health care and systems related challenges, and patient and family related challenges more specifically, regarding pediatric patients, the study had indicated that age, treatment refusal, knowledge about HIV status and treatment process, and depressive symptoms affect ART adherence. In order to define the challenges and identify the solutions, both HIV/AIDS counseling and testing aim at assisting clients/ patients to understand themselves and adjust effectively to life challenges and contribute meaningfully to the development of the society.
VCT is a key element to identifying HIV infected persons who could benefit from therapeutic interventions.

Effective and ethical counseling must recognize the impact of culture on a client's perception of the world. Counselors should take a holistic view of clients and their socio cultural background, including beliefs about HIV, sexual mores, traditional healing practices, gender inequalities, marriage practices (e.g., monogamy, polygamy), customs, and social practices. Therefore, the counselors should keep in mind that culture and tradition shape attitudes and beliefs, particularly regarding illness and death. On the other hand a counselor should refer clients to another counselor if differences of gender, race, ethnicity, religion, sexual orientation, disability, or socioeconomic status interfere in any way with counseling [18]. According to Assefa \& Kloos [12], the Provision of counseling services for ART patients do not exist in isolation from other health care services that must be collaborated with patients' counselors, physicians,' nurses, midwifes and other health care providers so that service providers together with ART patients are responsible directly to patients for every issue under treatment processes in order to have a positive outcome on ART patients' quality of life. The major challenges in the delivery of pediatric HIV services were related to the knowledge gap in pediatric HIV and the lack of counseling skills, as well as health system-related constraints.

Good health is an essential condition to meaningful national development. HIV/AIDS is a threat to life, thus individuals need to know their HIV status through testing. This is necessary because such a test helps to reduce transmission and involvement in risky sexual behaviors. It also promotes early treatment and adjustment [6]. Besides, good adherence to ART treatment increases better clinical outcomes which has been reported that the stepping-stones towards the achievement of long-term virus control among patients in developing countries are simplification of therapy, improvement of patient adherence and minimization of drug resistance [3].

According to Ritzenthaler [1], the global community indicated that ART as a key component of effective HIV/AIDS Programs. In their declaration of commitment, heads of state from 189 countries affirmed that prevention, care, support and treatment for those infected and affected by HIV/AIDS are mutually reinforcing elements of an effective response and must be integrated in a comprehensive approach to combat the epidemic".

In the late 1980s, as the epidemic surged and shifted from people with high-risk behavior to the Referral population, especially in developing countries, so that as Ritzenthaler [1] recommended that public health professionals should realize that they needed to better understand the causes of individual infection and the forces driving the pandemic that help health professionals to know how HIV transmission was linked to specific behavior such as having multiple sex partners, 
commercial sex, and injecting drug use, but they also realized that these behaviors were influenced by political, economic, social, cultural, and environmental factors-including poverty, military conflict, powerlessness, and gen when AIDS was first identified in the 1980s, public health officials assumed its spread could be halted by informing people how to protect themselves from infection and by safeguarding blood supplies [1].

According to the study report by Aldana, Pie-chule and $\mathrm{Al}-$ sabir, Client's satisfaction depends not only on service quality but also on patients' expectations. That is clients are more satisfied when services meet or exceed their expectations. If clients' expectations are low or if they have limited access to any services, they may be satisfied with relatively poor services. Furthermore, trust is the most important factor in the counselorclient relationship. This is because it enhances the relationship and improves the odds that an individual will act decisively on the information provided. On the other hand, in the situation given in the contexts of discrimination, ostracism, and personal recrimination that an individual diagnosed with HIV may have to face, it is all the more important to guarantee confidentiality.

Thus, client satisfaction has long been considered an important component when measuring health outcomes and quality of care. The rising strength of consumerism in society highlights the central role client's attitudes play in health planning and delivery. Furthermore, a satisfied client is more likely to develop a deeper and longer lasting relationship with their medical providers, leading to improved compliance, continuity of care, and ultimately better outcomes [14].

\section{Prospects of ART patients in the provision of counseling services}

Prospects in the provision of HIV counseling for ART patients in NRH were described as the ART patients' interest, wish, and belief towards the provision of counseling services that depend on ART patients satisfactions on the services provided. On the other hand, according to literatures, ART patient satisfaction depend on the context of the client's perceptions, service quality the client's and service provider relationship, and confidentiality and health outcomes.

Therefore, in this study the client's satisfaction in the services provided determined the client's prospects or interests towards the provision of counseling services that lead client to attend the regular follow up of counseling services. So, this study had tried to describe clients 'prospects toward in the provision of counseling services for ART patients and their participations of the treatments in NRH, chronic illness follow up clinic among ART patients.

\section{Materials and Methods}

\section{Study design}

As Pan Macmillan [19], emphasized that if the aim of qualitative research is to describe, identify or to understand how individuals perceive a particular issue, then qualitative methods are often appreciated, especially to describe people's experiences of health need, health care, accessing care and keeping healthy. Similarly, it helps to understand different perspectives such as those of professionals and patients, how experiences, attitudes and life circumstances affect health needs and behavior. As the knowledge of the researcher, no research was conducted with the same inquiry of this study topic since different researchers were conducted in international and national levels in the areas of adherence barriers, problems related with VCT, health services and health related problems due to HIV/AIDS rather than on challenges and prospects in the provision of counseling services for ART patents. Since qualitative research aimed to describe social phenomenon and behaviors using rich contextual data that emphasizes the subjective experiences of social actors, this type of data can be difficult to identify and describe using quantitative methods, particularly in using qualitative descriptive stages of research before hypotheses can be clearly articulated. The value of a qualitative perspective has gained favor in social behavioral health research and in HIV $\backslash$ AIDS research in particular, where any of the social phenomenon being studied are personal, intensely private, and sometimes illicit [20]. In addition to this the study participants (the clients) came from five woredas and one city administration from the nearby, this situation is important to conduct qualitative descriptive research from such participants since they have different background information and experience on the challenges and prospects in the provision of counseling services for ART patients. Therefore, this study involved a descriptive qualitative study design using qualitative data collection methods and described the challenges and prospects in the provision of counseling services for ART patients who were attending their follow up in NRH.

\section{Study area and populations}

This study was conducted in Nekemte Administration Town, the capital city of East Wollega zone in OROMIA, Nekemte Referral Hospital. Geographically, the hospital is located at $328 \mathrm{kms}$-far from Addis Ababa; the hospital is one of the public hospitals in the OROMIA. Approximately, 800,000-population is found in the hospital catchment area. Among different services given in the hospital, chronic illness follow up is one. Chronic illness clinic is one of the units of NRH. Currently the number of people living with HIV/AIDS (PLWHA) coming to the chronic illness clinic of $\mathrm{NRH}$ is estimated to be $\mathbf{5 8 4}$ who came from fife woredas (Wayu tuka wereda, Guto Gidda Woreda, Digga Woreda, Getema Woreda and Sasiga Woreda) and one city administration (Anger Gute) from the nearby . This clinic is currently running with 3 nurses, 2 general practitioners and 3 adherence supporters. Participants were selected from clients who were care of people living with $\mathrm{HIV}$ at NRH and service providers involved in the NRH chronic illness clinic, who were mainly low income individuals with different socio demographic backgrounds and experiences. Thus the source of population of this study were all ART patients 
attending NRH chronic illness follow up clinic and all service providers in the clinic during the study period.

\section{Sampling techniques}

Based on the inclusion criteria, this study involved a total of 48 study participants based on the characteristics of individual participants which consisted of 40 ART patients and 8 service providers in order to reflect the diversity and breadth of the sample population. The reason why the researcher involved such a large number of study participants was to limit the redundancy of data from the participants so that to increase data validity (which is congruent with assumption of as the study participants increase, the data validity also increase). This qualitative study used non-probability as it does not aim to produce a systematically representative sample or draw statistical inference. A criteria fulfilled ART patient, who came for his/her post counseling service, participated in the study. This trend continued till the researcher found the needed sample size. On the other hand, eight available service providers involved in the study. Therefore, due to the nature of qualitative research and the objective of the current study, the study employed non-probability sampling and purposive sampling techniques for the particular subgroups used to be involved to ensure sampling techniques on the basis of characteristics of study's participants (socio demographic characteristics, behaviors, attitudes, experiences)to reflect the diversity and breadth of sample population.

\section{Instruments of data collection}

This study had employed in depth interview using unstructured interview with study participants (ART patients who were attending follow up during the study periods and service providers who were giving counseling services for ART patients) and described the challenges and prospects in the provision of counseling services for ART patients. Unstructured interview is extremely important method to get in-depth information from the respondents. The interviewing was involved by asking questions and getting answers from participants on the study in the form of individual face-to-face interviews since development of rapport and dialogue is essential in unstructured interviews which prevent loosing important information. Moreover, as unstructured interviews are more flexible as questions can be adapted and changed depending on the respondents' answers, allow the respondents to talk in some depth, choosing their own words. This had helped the researcher develop a real sense a person understands of a situation to have increased data validity because it gives the interviewer the opportunity to probe for a deeper understanding, ask for clarification and allow the interviewee to steer the direction of the interview.

Therefore, the unstructured interview used in this study had included a matrix of questions options that were include behaviors' \experiences (questions about what participants does or has done aim to elicit behaviors', experiences, actions and activities that would have been describable had the researcher been present ); opinion\value questions (questions that need answers on respondents thought, opinion, the likes and dislikes in the provision of counseling); feelings \emotion questions ( that need adjective responses like anxious, happy, sad, afraid, intimated, confident during in the provision of counseling services for ART patients); knowledge questions ( that is about the respondents knowledge of the issues (about counseling processes, challenges and prospects in the provision of counseling services for ART patients).

\section{Methods of data analysis}

This study was mainly conducted using qualitative research that involved descriptive qualitative study design. Therefore, the qualitative data were gathered from study participants through unstructured interview using Afan Oromoo language that were further transcribed into Afan Oromo, the transcripts were translated into English, and the translated data were used for analyses. Furthermore, the translated data were identified line by line, summarized and obtained in Referral senses, and then categorized into data based themes followed by careful description of the data in the narrative form using the actual words of the respondents' ( both clients and service providers) responses in illustrative or vivid words, which were used in quotes to avoid unnecessary repetition. Finally the interrelation ships of the major challenges, common challenges, clients satisfaction on the HIV counseling services provided and the client's prospects or interests toward the provision of counseling services and participation of the clients in their treatments were identified and described, then results based conceptual frame works were developed. Through these activities, to limit the bias in interpretation, the researcher used constant comparison after each steps and among each steps results.

\section{Results}

\section{Socio-demographic characteristics of the respondents}

Here, the researcher presented the socio-demographic characteristics of both ART clients and service providers. As the ART clients responses indicated, most of them were come from country side (rural areas), aged 35 and above and who were married having discordant problem (one of the couple was either HIV positive or HIV negative); and others were divorced due to HIV /AIDS, widowed (lost their husbands or wives) due to HIV/AIDS. All of ART patients didn't take VCT due to serious sickness and they came by their own to have TB test, pregnancy check-up and due to lose of either husbands or wives in HIV/ AIDS cases. Besides, most of the study ART patients were under $12+2$ educational achievement (that is most of them were 7-10 grade level, very few from grade 8-10 and one participants was with $12+2$ education level). Almost all are economically poor, most had children among that some were with HIV positive who are attending their treatment, and some of the females were attending their treatment in a secretive way due to their 
husbands' involuntariness to attend the treatment. Almost all females were house holders, and almost all female clients were wives of social worker husbands, and few of them were waiters. Most of males' clients were from Derge military members and social workers, and all ART participants said as they have social influence. While service providers background information data indicated that most of the service providers were having medical professions who have training in HIV counseling from three weeks to two months given by NGOs, three of the service providers were adherents supporters who are living with HIV and taken training on HIV counseling but they have little education status (grade six to ten ) and very few are with lack of experiences for their profession's and had taken no training for HIV /AIDS counseling.

\section{Challenges and prospects in individual's regular practices in the provision of counseling services for ART patients}

Under this subcategory the researcher has got almost all clients' descriptions showed that distance and transport problem which resulted in time and money problems, economical problem, social and psychological problems, social influences related with discrimination, marginalization, stigma, lack of counseling rooms for one to one counseling problems have been found. Some study participants added that signs and symptoms due to the virus and medication side effects, problems due to discordant and the influence of males over females were indicated. Besides, the service providers responses indicated that misunderstanding of service providers about their clients emotional, psychological and personal, social and economic problems due to lack of training and experiences that resulted in similar and non-systematic advice mainly addressed. Furthermore, some service providers added the challenges as lack of awareness, knowledge and skills to identify and to treat specific problems of clients that might resulted from work shifting of well trained and experienced service providers and absence of professional counselors; they added that resistance of the client to take the reality and to go to the counseling rooms and workloads together with referral problems were another challenges. On the other hand, very few service providers described the challenges as males influence on females to accept, attend and follow up their treatment voluntarily; and lack of counseling rooms that result in privacy and confidentiality problems.

\section{Response of study participants in relation to the provision of counseling services}

Regarding the above idea, both respondents (client and service providers) responses indicated that they all think very positive in the provision of counseling services for ART patients if the problems described were solved. This implies that if the challenges in the provision of HIV counseling decreased and \or solved individual clients has positive thoughts towards counseling and on the effectiveness of counseling.

\section{Study participants' opinions on the challenges of the provision of counseling service}

Almost all of the ART patients described their unfavorable approach or opinion towards the provision of counseling services on the basis that it didn't help them solve their faced problems due to HIV/AIDS. These study participants would have positive opinion on counseling services if it helped them solve their psychological difficulties. So, ART patients would like to be benefited from the existing counseling provision if it was given with good advices and information to the extent that helped them to know what they hadn't, if nutrition was given with medication, if privacy and confidentiality was assured during the counseling process. Still very few respondents added some opinions regarding the challenges of counseling provision such as unfavorable approach of husbands and the family misunderstanding of HIV counseling, discordant, problems related with distance and transport, and counseling room's limitations. On the other hand, most of service providers had shared opinions on the challenges of ART counseling provision were included "no special attentions and approaches were given by different organizations (i.e. to solve the clients financial problems and social influences), awareness problem regarding the importance of HIV counseling provisions among the clients and societies, and almost no ART counseling training on up to date information regarding the changing needs and problems of the clients. Still very few service providers expressed their opinions that there was counseling skill gap, ART client's resistance to accept the nature and situation of HIV/AIDS and its related issues through counseling services.

\section{Expectations of study participants from the provision of counseling service}

The responses of most of the study clients, to the question of what would you like to see in the provision of counseling services?, in order to attend the regular counseling, first solution shall be given to our problems such as medication side effects, distance and transport problems, medication interruption, economic problems. In addition, some clients responses showed that, by considering their day to day life situations and for up to date information, they would like counseling being given by phones and in nearby clinics. Some respondents added that they expected counseling helped them on the way how to live long and proper lives, how to keep and run quality life and how to improve their self-care practices. Few clients responded that they would like counseling if it created good awareness among family members and community regarding the importance of HIV counseling and its importance in helping individuals deal with daily life difficulties, how a person living with HIV could secure good living style. Besides, very few clients added that they wanted ARV medication given for three months at one day through injection.

On the other hand, in counseling, almost all service providers would like to have enough materials for counseling and for flyers 
to be given to ART patients and the community, good coordination with ARV drug prescribers on sharing details of individual patients, and giving counseling services in nearby clinics or health centers. In addition, most service providers responded that they would like to get continuous training on ART patient counseling in order to develop up-to-date knowledge and skills in the identification, understanding and treating individuals with their specific problems. Still a very few service providers would like if VCT was given for the clients before counseling, to have organized counseling room for assuring privacy and confidentiality issues, and if professional counselors employed so that professional work shift problem might be minimized. From the above descriptions both respondents description implied that lack of awareness, knowledge and skill, poor service provision due to lack of training for service providers, absence of professional counselors, economic, social and personal problems, problem related with accessibility (distance and transport), and unavailability private and confidential counseling rooms are among major problems of both service provider and clients of counseling.

\section{Feelings of study participants on challenges and prospects of counseling provision}

According to the client respondents, individuals feeling in the provision of counseling services for ART patients indicated that, most of the clients responded as they had negative feelings due to unsolved problems that they described including emotional, personal, social psychological and economic problems. Besides, few of the respondents responded that they have negative feelings due to lack of social supports, lack of counseling services in their nearby clinic, due to distance, transport and economic problems. Moreover, very few respondents described as they felt negative in the services provided due to their discordant problems and males influences on females that didn't solved by counseling, and still very few described their negative feelings on the services provided as it was not solved their personal problems that limited their quality lives. According to the service providers, most of them described their feeling that they felt sorry due to lack of training and experiences which made them to be ambiguous while counseling their clients that made them give similar information for different client with different problems. Some described that due to knowledge gaps by the society and the client about the importance of counseling, the clients were not ready to accept the results. Few service providers added that they were not satisfied with the service they provided because of lack of counseling rooms that resulted in problems in privacy and confidentiality. The implication indicated that the study participants had negative feelings on the prospects of counseling services for ART patient due to unsolved such problems, described under the respondent feelings, as emotional, social, psychological and economic problem, lack of training, absence of professionals counselors, lack of awareness on importance of counseling, absence of counseling services in nearby clinics, lack social supports for ART patients, absence of counseling rooms, discordant problems, husband influences on wife were problems led to dropouts, interruptions and retentions in both medication and counseling treatments that might in turn resulted in health related problems due to medication side effects, the virus and lack of information which also resulted in poor self-care practices which also might led the respondents to have negative perception in the provision of counseling services for ART patients.

\section{Knowledge of the study participants on the way that how they came to ART treatments:}

According to the clients, all of them knew their ways that how they came to ART treatment, almost all of them described that they come to the treatment by their own due to serious illness and sickness, TB testing, some come due to either their husbands \wives were died due to HIV/AIDS, still some came to have pregnancy checkups.Still very few came due to their friends advice, however all hadn `t have VCT and they have no knowledge on the importance of VCT and all of them described that they were happy due to medication that made them recover from such serious illness and bed sick and provision of medications which is free from fee but they were not happy in counseling service provided due to their unsolved problems in counseling service provision. While service providers responses indicated that although all of them were not professional counselors most were trained from three weeks to two months by NGOs, few have absence of training and experiences, and adherent supporters who had living with HIV and took training for counseling for people living with HIV but they lack educational background (from grade six to ten plus two). All service providers are happy in providing counseling but they need further trainings and educations that lack training, experiences and education but they were not happy on challenges that they met during counseling. The above data implied that both respondents descriptions showed that they all have the knowledge on the way how they came to ART treatment and they all were happy in participating the treatment, however; their happiness depended on different contexts for example the client were happy due to medication rather than counseling, service providers were happy due to being counselors is human helping process but lack of education, training and experiences that made them unhappy.

\section{Major problems in provision of counseling services for ART patients}

According to all study participants responses, the major problems described include lack of awareness and skills by service providers on counseling provision, lack of practice by ART patients on their personal, self and living styles, lack of decentralization, problems related in societies and families, absence of professional counselors, absence of NGOs, absence of support from societies, and local governments, lack of organized economic opportunities, limitation of counseling rooms for one to one counseling, social influences such as discrimination ,marginalization and stigmatization, lack of awareness, 
knowledge and skills on HIV counseling; interruption and dropouts of both treatments and medications .Transport and distance problems, low education levels of both ART clients and adherent supporters ,marital complication due to HIV $\backslash$ AIDS, resistance by both the client and society, lack of training and experiences by the service providers due to work shifting; and work load with lack of linkage for service providers.

\section{Study participant's expressions about their satisfaction in the provision of counseling services provided}

The researcher described both ART patients and service providers' responses about their satisfaction in the provision of counseling as below: According to client respondents, almost all clients described that they were satisfied by getting medication free from fee, for they recovered from serious illness and bed sick due to medications. However, all were in doubt as they were satisfied in the provision of counseling because of the problems they experienced in the provision of counseling. Similarly some service providers expressed their clients satisfactions that include good relationships and confidentiality but all service providers didn't expressed their clients satisfaction in the context of counseling provision, however; all service providers including adherent supporter agree that all clients were satisfied when they were with adherent supports that they become the models for them. All adherent supports described their client satisfaction that they express their client satisfaction in the context of the client emotional contexts that is the clients were happy with them and telling them everything when they met with adherent supporters for the clients see the adherent supports as models and the client exchange the phone and make communication with them even after the client went to their home , and they make continuous communication through phone about problems they met at home and in the environment.

As the above items (items 1 to 9) data summary indicated that all ART patients have knowledge on how they came to the treatments, on the problems that they faced in their ways to counseling services and during counseling provision but they have lack of awareness and knowledge on HIV/AIDS, signs, causes, symptoms, preventive measures in clouding self-care practices, and lack of knowledge on counseling processes as well as the importance of HIV counseling services .On the other hand, all service providers said that they have knowledge on how they became to service providers, on the problems they had faced in the provision of counseling service, but they have lack of awareness, knowledge and skills on identifying, understanding and treating the ART patients' personal, social, emotional, psychological problems they had faced in the provision of counseling services for their clients since in the proviso they were not professional counselors but some were trained on HIV counseling, some were trained but lack of experiences, some were trained but long time ago, some were neither trained nor experienced and still some specially adherent supporters were trained a long time ago but with low education achievements.

To the end, both respondents (clients and service providers) described their opinions, feelings, emotions and the changes that they wanted to see to happen in the provision on of counseling services indicated that they would have positive opinions, feelings and emotions if the problems that were either directly or in directly challenging the provision of counseling services for ART clients would be solved or minimized. These implied that the problems described by study participants could determine their satisfaction on the service provided that in turn determine the client thoughts \interests to world the provision of counseling services and in participations of their treatments.

\section{Discussions}

In this section, the researcher gave meaning to the study findings and discussed them with previous study results.

\section{Study findings in relation to previous study results}

No international, national, and local researches were made with same inquiry of this study. In addition to this, differences due to study periods, study area, study type, and study participants, not all literatures but some were related with the findings of this study either directly or indirectly related with ART issues thereby contributed to this study. So, the researcher discussed these gaps in detail as follows:

In this study, the challenges for counseling provision for ART patients determining the ART clients' satisfaction on the services provided and thereby determining their prospects or thoughts toward the provision of counseling services and participating in the treatment services were transport costs, hunger, stigma, long waiting, medication side effects, and lack of appropriate counseling. This finding is also well supported by previous study results done in Botswana Tanzania and Uganda. However, user fees were not the challenges counseling services in this study. The difference might be that in Ethiopia settings, ART patients get treatment services with free of payments.

Other challenges, in the provision of counseling services for ART patents, were lack of resources such as distance and transport accessibility, limitation of counseling rooms, lack of decentralization, and lack of appropriate awareness creation materials and activities; and work shifting of professionals. This finding was almost similar with the study done in Ethiopia by Assefay et al, [13]. In a quantitative study conducted by Rosen \& Gill [3], variables such as sex, marital status, religions, ethnicity and place of residence had no association with HRQOL of the ART patients. However, in current study, despite religion and ethnicity which had no influence on HRQOL of ART patients, challenges such as sex (identified as males influence in females not be have voluntary HIV counseling and testing and to have attended their treatment) marital status (marital complication due to HIV/AIDS that resulted in divorce, discordant lose of the couple's due to HIVAIDS, one of the couple' knew their HIV status and the other 
not, one of the couple's attending the treatment and the other not) which might resulted in disorganized families that had led to economic dependence, loss of confidence , loss of potential. In addition ,place of residence (especially rural clients who lacked decentralization for counseling services in their nearby clinic, distance and transport problems that might resulted in time and money problems that had also led to interruption, drop outs and retention in both treatment and medication that resulted in health related problems .

One of the observations of this study finding was that, at patient centered health care services, the first challenges were identifying and meeting the changing needs of the ART patients. This was similar to the observation made by WHO [10]. Besides, WHO suggested that service providers need to ensure that patients can access counseling and medicines easily at a time and place of their own choose. This could empower patients engaging herein dialogue to communicate knowledge and enabling them manages their health and treatment. However, this suggestion was not applied in the study area. This could be due to the challenges such as service-related problems, clients related, service provider related and socio-demographic related problems rather than service providers alone. In this study, knowledge, attitude and belief regarding HIV/AIDS were found to influence the decision to accept or reject VCT and provision of counseling. This result is supported by previous study [3]. However, in this study, lack of awareness, male influences on female and service providers related challenges were found to challenge VCT and provision of HIV counseling. Besides, socio demographic characteristic, service-related problems, service providers related problems and clients and social related problems also to challenge HIV voluntary counseling and testing.

On the other hand, role of home care, and community oriented care services which were described under current study as family related problems and lack of social supports, respectively, and lack of integration among service providers were found to be problems of ART patient's health care and effective HIV counseling. This supported report of global HIV/AIDS (2004). Another problems found by the researcher were include servicerelated problems (such as lack of management and evaluation for day to day experienced problems lack of counseling rooms for one to one counseling work shift of professional service providers. This led the patients felt difficulty of privacy and confidentiality which might led the clients to develop poor satisfaction on counseling services provided that might in turn challenged the clients need, interest or thoughts toward the ART treatments and participating in the treatments. With instance, for the better health care service of ART patients, the researcher recommended that it is better to give the first hand emphases on professional quality, training and education of professional service providers and then integration these service providers, so that these shall be the important steps to assign service providers and help them have quality integration among them thereby to provide adequate HIV counseling. Other counseling services challenges found by this study include negative attitude, lack of awareness and knowledge gaps, and negative social fluencies like discrimination and stigma. This is supported by previous research findings [12].

As WHO [2] defined that HIV /AIDS counseling involves educating client or a group of clients on the control, management and prevention of HIV /AIDS. This WHO's definition is very useful for the provision of effective HIV counseling that help both service providers and clients in the provision of counseling service for ART patients. However, this definition was not applied in this study area since there were challenges related with the client's awareness, knowledge, and attitude towards counseling, and lack of adequate knowledge and skills of service providers in the provision of counseling service. In addition, this study identified problems that had related with clients such as lack of awareness and knowledge gaps on sings, symptoms, causes, cares and counseling process; negative attitude/or beliefs (such as fear of being HIV positive, society and family due to the disease). This is similar with previous study results [12]. Service providers related problems including lack of training and experience on HIV counseling (since most service providers were from medical professionals), absence of professional counselors that resulted in provision of similar information ( such as non-systematic advice, education and teaching) for clients with different problems that might hindered effective counseling activities such as identifying, understanding and treating ART patients with emotional and psychological problems. Besides, the current study identified and described challenges in the provision of counseling service for ART patients as work shift of well trained and experienced service providers, counseling relationship between experienced clients and new service providers which was characterized by reintroduction, re beginning with the new service provider which resulted in ineffective counseling the problems resulted from privacy and confidentiality. Besides, especially the study participants were with low education levels, they couldn't identify the names of medication unless they brought the containers of medication to their new service providers. In this case most clients had provided different medications due to poor prescription of drugs that had worsened medication side effects. Above all these clients felt discomfort to have intimation and discuss their problems with the new service providers that might resulted from poor relationship due to service providers related problems and service related problems (such as limitation of counseling rooms that might led to poor reception of the clients ). However, these findings are contradicted with WHO's [2] explanation of what counseling service as helping (which is not giving advice) relationships to help a person help himself/ herself deal with an aspect of his life that causes discomfort (problem/concern). Besides, counseling is the ground whereby the client feels comfort with service provider accepts and becomes "self".

Recommendations from all study participants had forwarded (such as problems related to service providers, service related, 
social influences, marital complication medications side effects) that might need to be solved. In addition, all study participants recommended that they would like to see if awareness creation materials and activities will be provided, if social influences will be solved, if the problems related with distance ,transport, lack of decentralized clinics, problems related with privacy and confidentiality and poor relation of the clients within the families and the society and between the clients service providers ,limitation of counseling rooms. They also recommended that problems related with service providers and the services will be solved thereby their personal, social, emotional, psychological and economic problems will be minimized with the help of counseling services that will make them to be satisfied in the service provided and to have positive thoughts or prospects toward the provision of counseling services and participating in the treatments thereby attending the treatments regularly. The above recommendations were either directly or in directly similar with the recommendations forwarded by Okpoto [11], Abishu [18], Global AIDS, Assefay et al, [13], WHO [10] and Rzenthaler [1].

This study found that ART patients were not satisfied with the provided services because of the challenges in the provision of counseling services described as long waiting time(due to distance and transport problems ,limitations of counseling rooms, fear of social influences),poor or non-systematic flow of information resulted from absence of professional counselors ,work shift of experienced and trained service providers ,lack of education for both ART clients and adherent supporters; privacy and confidentiality; and poor relationship between ART clients and service providers. This is supported by Al Dana \& Lochoro $[14,16]$ saying that client's satisfaction depend on service quality and client's expectation on services and the clients social relationships wellbeing which guaranteed confidentiality and counseling-client relationships, health outcomes and quality of care.

Therefore, the above described challenges of counseling provisions among the study ART patients imply that poor clientcounselor relationship, poor client-counselor information flow, privacy and confidentiality related problems, and long waiting time that might resulted in negative service satisfactions, that might lead to negative prospects $\backslash$ thoughts of the clients toward medication and counseling provisions and participation in the treatments. Therefore, this study suggested activities on the control, management, and prevention of HIV/AIDS through HIV counseling, identification of client's holistic conditions and employing skilled professional counselors should be guaranteed [21].

To sum up, as human being is a social being, an individual problem have either social grounds or social problems have an individual related grounds either directly or indirectly due to the nature of human problems that have a tendency to defuse from one individuals to another or via versa uncles properly identified and treated. HIV/AIDS is among human beings problems that can be treated through medications and different methods of treatments including HIV counseling [22].

Effective provision of counseling services for ART patients must consider the clients socio demographic characteristics related problems, clients related problems , the service providers related problems, the service related problems and psychosocial related problems together with their common inter relationships including long waiting time, poor relationships among and/ or between clients and the families, clients and the society in the immediate and surrounding environments in Referral and between the clients and the service providers during the treatment process in particular; privacy and confidentiality related problems and problems related with poor or nonsystematic flow of information by the family and the society and by the clients and service providers in particular during the provision of counseling [23].

Therefore, such provision of counseling services for ART patients might resulted in the clients satisfaction on the services provided that might led the clients to have positive prospects /beliefs/ interests in participating in both medication and treatments. Thus the satisfied clients have positive prospects toward participating in both medication and treatment that resulted in reducing interruptions, dropouts and retentions in both medications and treatments (that is, the satisfied clients keep regular follow-up of both medications and treatments) that in turn resulted in describing the clients health related problems resulted from medication side effects, the virus and non-systematic information provided in the families, in the society and during counseling provision. Decreasing these kinds of health related problems resulted in increasing better self-care practices and thereby increasing better health related quality of life. On the other hand, reducing problems related to poor selfcare practices led to reducing problems related to poor health related quality of life, thus better self-care practices resulted in better health related quality of life that decrease or limit new HIV transmission, bed sickness and/or death and complication of marriage on ART patients due to HIV/AIDS that decrease disorganized families (orphans, single parents) and increase well organized families, societies and economic conditions. Based on the above described problems implication, which were chained with their cause effect relationships, the researcher constructed the following data based alternative conceptual frame works.

\section{Conclusion}

In the study results, challenges and prospects in the provision of counseling services for ART patients in NRH were identified and described in words which were developed with the actual responses of study participants. The challenges were found in the study as the major challenges and common challenges that might challenge the provision of counseling services for ART patients in NRH. In the contexts of these challenges, the clients' 
satisfaction on the services provided and the client's prospects/ interests or beliefs toward the provision of counseling services and participation of their treatments were identified and described with their consequences in the results chains. Accordingly, the major challenges in the provision of counseling services for ART patients in NRH included socio demographic characteristics related, clients related, service providers related, service related and psychosocial related problems that were either directly or indirectly challenging the provision of counseling services for ART patient. In addition ,the major challenges might resulted in common challenges that included poor relationships between the clients and service providers (resulted from poor reception, work shift of service providers, counseling room limitation, social influences);poor information flow (due to lack of ARV ,poor drug prescription, non-systematic advice, lack of active participation between clients and service providers similar information for different clients);privacy and confidentiality problems (due to work shift of service providers ,social influences, counseling room limitations, lack of keeping the secret after counseling); and longtime waiting resulted from service related, social influences (waiting for long time not be seen to enter or to be out of counseling room, distance and transport problems).These common challenges might directly challenge the provision of counseling services for ART patients in NRH.

Due to the major challenges in general and common challenges in particular the study ART patients were not satisfied in the HIV counseling services provided that might led the clients to develop negative prospects/interests toward the provision of counseling services as well as their participation in the treatment services thereby they might developed interruptions, dropout and retentions of both medications and treatments which might resulted in medication side effects resulted from the virus, and lack of effective information for selfcare practices such conditions might developed health related problems (such as poor self-perception/self-esteem, negative emotional and psychological conditions including helplessness and hopelessness, anger and aggression, worries and sadness, lose, fear, as well as revenge development) on ART patients that were resulted from lack of social supports (in the contexts of economic ,emotional and psychological ),social influences (for instance, discrimination, stigma, marginalization ,and finger pointing) that might led to problems related with privacy and confidentiality, relationship, information exchange and selfcare-so that such health related problems might resulted in new HIV transmission, illness/sickness and/or death of ART patients in HIV/AIDS case that in turn led to disorganized ART clients martial and familial conditions that may result in problems in economic, social and development conditions.

Based on the findings the researcher recommends the following: a) The service providers in NRH may benefits from additional training on HIV counseling and adherent supporters, especially, may benefit from both training and upgrading their educational levels there by all HIV counseling service providers can develop awareness, knowledge and skills on HIV counseling processes.

b) Nekemte referral hospital may benefit and provide effective HIV counseling services if assign professional counselors, limit experienced and trained service providers work shift, solve service related problems such as limitations of counseling rooms for these might resulted in privacy and confidentially problems, poor relationship of clients and service providers, poor information provision, and long waiting time during the provision of counseling services for ART patients.

c) ART patents in NRH may benefit from marriage and family counseling as well as psycho social counseling since these areas of problems might affected the clients personal ,social ,emotional, psychological and economic conditions in the contexts of the clients immediate and the surrounding living conditions as well as their ways to the treatments and during their treatments.

d) Community integrated awareness creation activities should be created on HIV/AIDS issues in Referral and on the importance of HIV treatments in particular In addition ,well cooperated and organized social integrations should be created and award to support the ART patents in economic, emotional, social, and psychological contexts by involving ART patients in activities at home, community and social levels.

e) Further studies should explore the pattern of HIV counseling in settings, So that further studies should involve different data collection instruments including observation and focus group discussion, considering both the major challenges and common challenge in the contexts of identifying, understanding and treating the clients personal, social, physical, emotional, psychological and economic conditions.

\section{References}

1. Ritzenthaler (2005) Delivering Antiretroviral therapy in Renounceconstrained-setting.

2. WHO (2003) Global HIV/AIDS report. A joint WHO/UNIADS report. Geneva, Switzerland.

3. Rosen S, Fox MP, Gill CJ (2007) Patient retention in antiretroviral therapy programs in Sub-Sahara Africana systematic review. PLoS Med 4(10): e298.

4. Mendoza Aldana J, Piechulek H, Al-Sabir A (2001) Client satisfaction and quality of health care in rural Bangladesh. Bull World Health Organ 79(6): 512-517.

5. Holmberg SD (1998) Declining morbidity and mortality among patients with advanced human Immune deficiency virus infection HIV Outpatient Study Investigators. N Engl J Med 338(13): 853-860. 
6. Assefa Y, Van Damme W, Mariam DH, Kloos H (2010) Toward Universal Access to HIV Counseling and Testing and Antiretroviral Treatment in Ethiopia: Looking Beyond HIV Testing and ART Initiation. AIDS Patient Care and ST 24(8): 521-525.

7. Chan KC, Wong KH, Lee SS (2006) Universal decline in mortality in patients with advanced HIV-1 disease in various demographic subpopulations after the Introduction of HAART in Hong Kong. HIV Med 7(3): 186-192.

8. UNAIDS (2012) UNAIDS Report on the global AIDS epidemic Geneva, Switzerland.

9. WHO (2012) Retention in HIV programmers: Defining the challenges and identifying.

10. WHO (2013) Consolidated guidelines on the use of antiretroviral drugs for treating and preventing HIV infection: recommendations for a public health approach. Geneva, Switzerland.

11. Okpoto RI (2009) Attitude of university of Ilorin undergraduates towards voluntary HIV/AIDS Counseling and testing. An unpublished B.Ed project Submitted to the Department of Counselor Education, University of Ilorin, Nigeria.

12. Assefa Y, Kloos H (2005) The public health approach to ART service scale-up in Ethiopia: the first two years of free ART, 2005-2007. Ethiop Med J46(4): 401-406

13. Assefa Y, Jerene D, Lulseged S, Ooms G, Van Damme W (2009) Rapid Scale-up of Antiretroviral Treatment in Ethiopia: Successes and System-wide Effects. PLoS Med 6(4): e1000056.
14. Lochoro P (2004) Measuring client satisfaction in UCMB health institution. Health policy.

15. UNAIDS (2013) UNAIDS Report on the global AIDS epidemic |. Geneva, Switzerland.

16. Dan H, Longo A, Fauci S (2005) The Human Retroviruses. Harrison principles of internal Medicine (16 ${ }^{\text {th }}$ edn).

17. Sibhatu, Ayalu (2011) Adherence to Antiretroviral Therapy in Sub Saharan Africa; Challenges and Prospects.

18. Abishu G (2011) Ethiopian Counseling Tradition in reference to Modern. Western counseling System. Extracted from PHD Dissertation Paper, Unpublished.

19. Pan Macmillan (1979) Douglas Adams, the Hit -Hiker's guide to the Galaxy: London.

20. Rotchford AP, Rotchford KM, Mthethwa LP, Johnson GJ (2002) Reasons for poor cataract surgery uptake-a qualitative research study in rural south Africa. Trop Med Int Health 7(3): 288-292.

21. WHO (2002) Health organization? 3 by 5 Global initiatives providing Anti-retroviral therapy. Treating million people with HIV/AIDS in developing country. Geneva, Switzerland.

22. Stephen A Margolis, Manzouqi AT (2007) Client satisfaction with primary health care service in the United Arab Emirates. Journal in Health care Intervention in an emergency pediatric unit 29: 241-249.

23. USAID (2011) Country profile, USAID, Ethiopia. Ethiopia riverside.

This work is licensed under Creative Commons Attribution 4.0 License

DOI: 10.19080/ARGH.2019.14.555881 